PREPARED FOR THE U.S. DEPARTMENT OF ENERGY, UNDER CONTRACT DE-AC02-76CH03073

PPPL-3927

PPPL-3927

UC-70

\author{
Hamiltonian Description \\ of Convective-cell Generation \\ by \\ J.A. Krommes and R.A. Kolesnikov
}

March 2004

NM|

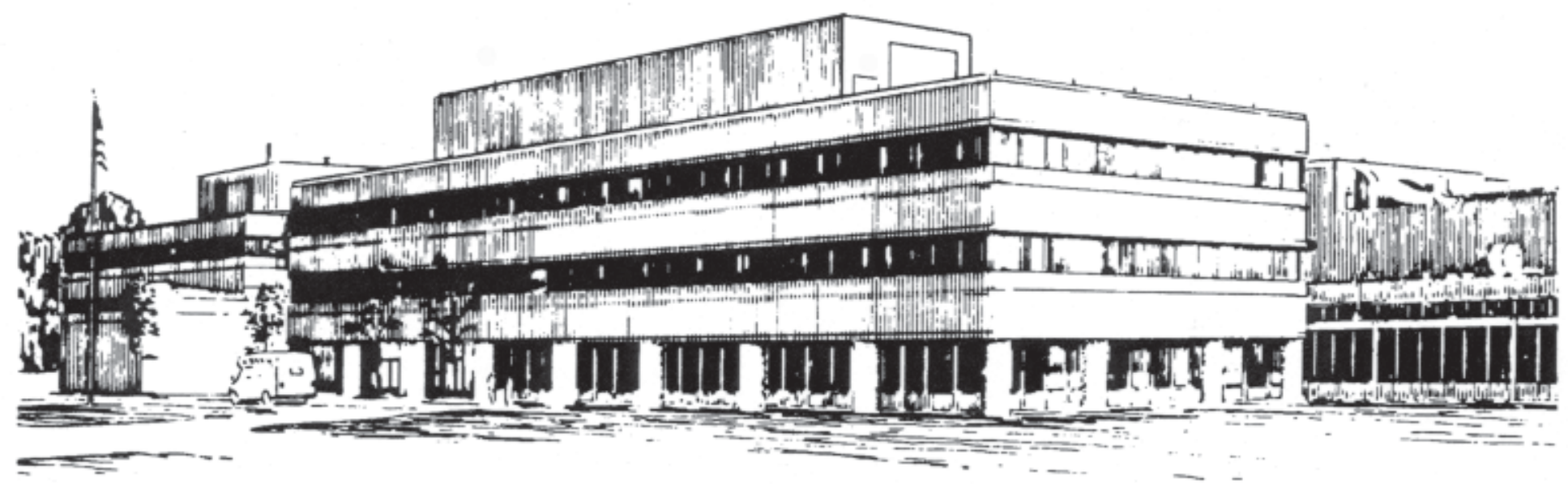

PRINCETON PLASMA PHYSICS LABORATORY PRINCETON UNIVERSITY, PRINCETON, NEW JERSEY 


\section{PPPL Reports Disclaimer}

This report was prepared as an account of work sponsored by an agency of the United States Government. Neither the United States Government nor any agency thereof, nor any of their employees, makes any warranty, express or implied, or assumes any legal liability or responsibility for the accuracy, completeness, or usefulness of any information, apparatus, product, or process disclosed, or represents that its use would not infringe privately owned rights. Reference herein to any specific commercial product, process, or service by trade name, trademark, manufacturer, or otherwise, does not necessarily constitute or imply its endorsement, recommendation, or favoring by the United States Government or any agency thereof. The views and opinions of authors expressed herein do not necessarily state or reflect those of the United States Government or any agency thereof.

\section{Availability}

This report is posted on the U.S. Department of Energy's Princeton Plasma Physics Laboratory Publications and Reports web site in Fiscal Year 2004. The home page for PPPL Reports and Publications is: http://www.pppl.gov/pub_report/

DOE and DOE Contractors can obtain copies of this report from:

U.S. Department of Energy

Office of Scientific and Technical Information

DOE Technical Information Services (DTIS)

P.O. Box 62

Oak Ridge, TN 37831

Telephone: (865) 576-8401

Fax: (865) 576-5728

Email: reports@adonis.osti.gov

This report is available to the general public from:

National Technical Information Service

U.S. Department of Commerce

5285 Port Royal Road

Springfield, VA 22161

Telephone: $1-800-553-6847$ or

(703) $605-6000$

Fax: (703) 321-8547

Internet: http://www.ntis.gov/ordering.htm 


\title{
Hamiltonian description of convective-cell generation
}

\author{
J. A. Krommes and R. A. Kolesnikov* \\ Plasma Physics Laboratory, Princeton University, P.O. Box 451, Princeton, New Jersey 08543
}

(Dated: February 26, 2004)

\begin{abstract}
The nonlinear statistical growth rate $\gamma_{\boldsymbol{q}}$ for convective cells driven by drift-wave (DW) interactions is studied with the aid of a covariant Hamiltonian formalism for the gyrofluid nonlinearities. A statistical energy theorem is proven that relates $\gamma_{\boldsymbol{q}}$ to a second functional tensor derivative of the DW energy. This generalizes to a wide class of systems of coupled partial differential equations a previous result for scalar dynamics. Applications to (i) electrostatic ion-temperature-gradient-driven modes at small ion temperature, and (ii) weakly electromagnetic collisional DW's are noted.
\end{abstract}

We present a covariant Hamiltonian derivation of the nonlinear statistical growth rate $\gamma_{\boldsymbol{q}}$ of long-wavelength convective cells [CC's; wavevector $\boldsymbol{q}=\left(q_{x}, q_{y}, 0\right)$ ] driven by short-wavelength drift waves [DW's; wavevector $\boldsymbol{k}=$ $\left.\left(k_{x}, k_{y}, k_{z} \neq 0\right)\right]$. The concise result encompasses all previously known results for dynamics governed by scalar field equations and also applies to systems of coupled partial differential equations (PDE's) that are very difficult to treat by standard procedures. We focus on the general formalism, but do comment briefly on the calculation of $\gamma_{\boldsymbol{q}}$ for CC's driven by (i) electrostatic ion-temperaturegradient-driven (ITG) fluctuations, and (ii) weakly electromagnetic collisional DW's.

The original attempt at a workable formalism for the calculation of $\gamma_{\boldsymbol{q}}$ was made for the special case of zonal flows $\left[\boldsymbol{q}=\left(q_{x}, 0,0\right)\right]$ by Diamond et al. ${ }^{1}{ }^{1}$ who attempted to use WKB methods to calculate the modulational effect on the DW's. Krommes and $\mathrm{Kim}^{2}$ (KK) revisited, critiqued, and generalized those calculations and showed how the ideas were related to Markovian closures ${ }^{3}$ and statistical field theory. ${ }^{2-4}$ For the instructive limit of cold ions, their result was the most general to date, as their expression for $\gamma_{\boldsymbol{q}}$ pertained to all convective cells, not just zonal flows. [Apparently unaware of the work in Ref. 2, Kim and Diamond ${ }^{5}$ (KD) later published a different expression for the cold-ion $\gamma_{\boldsymbol{q}}$. However, a conceptual error in that calculation was identified by Krommes; ${ }^{6}$ when corrected, the calculation of KD was brought into agreement with the earlier one of KK.]

In all calculations to date, a scalar wave kinetic equation (WKE) was employed for the DW's and $\gamma_{\boldsymbol{q}}$ was found to depend on the spectral density $\mathcal{Z}_{\boldsymbol{k}}$ of a conserved quantity $\mathcal{Z}$ that we note below is a Casimir invariant. However, most applications are naturally formulated in terms of systems of coupled fields and require the use of a tensor spectral balance equation for the correlation matrix $\mathrm{C}$. There may be multiple Casimirs, all of which constrain the dynamics but are fewer in number than the independent elements of C. Preferentially singling out individual components of the tensor WKE leads to tedious algebra that obscures the general structure. What is needed is a formulation that treats all components of the WKE on equal footing. By focusing on the Hamiltonian structure of the nonlinear interactions and expressing the resulting dynamics in a (nonrelativistic) covariant way, we have obtained such a description. In general, $\gamma_{\boldsymbol{q}}$ no longer depends merely on a scalar $\mathcal{Z}_{\boldsymbol{k}}$ even for a single Casimir, although its form is constrained by Casimir conservation.

Since the work of Morrison, Greene, and others in the 1980's (reviewed in Ref. 7), it has been recognized that nondissipative nonlinear systems frequently possess a (noncanonical) Hamiltonian structure. Therefore, we assume that we are given the coupled system of PDE's $\partial_{t} \psi^{i}=L_{j}^{i} \psi^{j}+\left\{\psi^{i}, \mathcal{H}\right\}$, where linear dynamics are described by the linear operator $L_{j}^{i}$ (independent of $\boldsymbol{\psi} \equiv$ $\left.\psi^{i}\right)$, summation over repeated indices is assumed, the Hamiltonian functional $\mathcal{H}[\boldsymbol{\psi}]$ is given, and $\{\cdot, \cdot\}$ is an appropriate Poisson-bracket operator. We assume that $\mathcal{H}$ has the form of a generalized kinetic energy, namely $\mathcal{H}[\psi]=\frac{1}{2} \overline{\psi^{i} \widehat{g}_{i j} \psi^{j}}$, where the overline denotes the integral over all space and $\widehat{\mathrm{g}}$ is a symmetric covariant matrix linear integral operator $[\widehat{g}=\widehat{g}(\nabla)]$ independent of both $\boldsymbol{\psi}$ and $t$. The key properties of the bracket are antisymmetry $(\{A, B\}=-\{B, A\})$ and the Jacobi identity $(\{\{A, B\}, C\}+\{\{B, C\}, A\}+\{\{C, A\}, B\}=0)$, where $A, B$, and $C$ are arbitrary functionals. We consider a noncanonical bracket of Lie-Poisson form, ${ }^{7}$

$$
\{A, B\}=\overline{S^{i j}\left[\frac{\delta A}{\delta \psi^{i}}, \frac{\delta B}{\delta \psi^{j}}\right]},
$$

where $\mathbf{S}$ is symmetric and linear in $\boldsymbol{\psi}\left(S^{i j}[\boldsymbol{\psi}]=S^{i j}{ }_{k} \psi^{k}\right)$ and $[\cdot, \cdot]$ denotes the $\boldsymbol{x}$-space Poisson bracket

$$
[A, B] \doteq\left(\partial_{x} A\right)\left(\partial_{y} B\right)-\left(\partial_{y} A\right)\left(\partial_{x} B\right)=\widehat{\boldsymbol{z}} \times \nabla A \cdot \nabla B
$$

( $\doteq$ denotes definition). The Jacobi identity is satisfied if $T^{i j k}{ }_{m} \doteq S^{i j}{ }_{l} S^{l k}{ }_{m}$ is fully symmetric in $i, j$, and $k(\forall m)$.

Although the above assumptions are restrictive, they encompass many physical systems, especially those that describe magnetized plasmas dominated by the essentially two-dimensional $\boldsymbol{E} \times \boldsymbol{B}$ nonlinearity. For example, as discussed later, the nonlinear dynamics of ITG modes at small $\tau \doteq T_{i} / T_{e}$ are described by the Hamiltonian (15) and the structure matrix (16b). This new result generalizes the known bracket structure of the 2D Euler equation for vorticity $\omega$, namely ${ }^{7} \partial_{t} \omega=\{\omega, \mathcal{H}\}$, where $\mathcal{H}[\omega]=\frac{1}{2} \overline{\omega\left(-\nabla^{-2}\right) \omega}$ and $\{A, B\}=\overline{\omega[\delta A / \delta \omega, \delta B / \delta \omega]}$.

Properties of the $\boldsymbol{x}$-space Poisson brackets include $[A, B]=-[B, A],[A, B C]=B[A, C]+[A, B] C$, and 
$\overline{A[B, C]}=\overline{[A, B] C}$. These results are useful in deriving conservation laws. For example, one proves that $\mathcal{H}$ is nonlinearly conserved as follows. From Eq. (1), the nonlinear dynamics can be written explicitly as

$$
\dot{\psi}^{i}=\left\{\psi^{i}, \mathcal{H}\right\}=-\left[S^{i j}, \delta \mathcal{H} / \delta \psi^{j}\right] .
$$

Then, upon noting that $\dot{\mathcal{H}}=\overline{\left(\delta \mathcal{H} / \delta \psi^{i}\right) \dot{\psi}^{i}}$, one has

$$
\dot{\mathcal{H}}=-\overline{\frac{\delta \mathcal{H}}{\delta \psi^{i}}\left[S^{i j}, \frac{\delta \mathcal{H}}{\delta \psi^{j}}\right]}=-\overline{S^{i j}\left[\frac{\delta \mathcal{H}}{\delta \psi^{j}}, \frac{\delta \mathcal{H}}{\delta \psi^{i}}\right]}=0,
$$

the last result following from the contraction of a symmetric and an antisymmetric form. Similarly, it is easy to show that when $S^{i j}{ }_{k}=S^{i k}{ }_{j}$ the invariant $\mathcal{Z} \doteq \frac{1}{2} \overline{\psi^{i} \psi^{i}}$ is also conserved. $\mathcal{Z}$ is a Casimir invariant, ${ }^{7}$ i.e., conserved for arbitrary Hamiltonian. The quantities discussed by Smolyakov and Diamond ${ }^{8}$ and KK as being conserved under modulation and identified as appropriate plasmon densities are special cases of this invariant. (Casimir invariants can exist even when $S^{i j}{ }_{k} \neq S^{i k}{ }_{j}$.)

According to Eq. (3), $\dot{\boldsymbol{\psi}}$ is determined by $\psi^{i}$ and $\widehat{\psi}_{i} \doteq \delta \mathcal{H} / \delta \psi^{i}=\widehat{g}_{i j} \psi^{j}$. Because $\widehat{\mathrm{g}}$ is symmetric, it can serve as a covariant metric tensor ${ }^{14}$ that lowers contravariant indices to covariant ones. Thus $\widehat{\boldsymbol{\psi}}$ is the covariant representation of $\boldsymbol{\psi}\left(\widehat{\psi}_{i}=\psi_{i}\right)$, and $\mathcal{H}$ can be written covariantly as $\mathcal{H}=\frac{1}{2} \overline{\psi^{i} \psi_{i}}$. Note that $\widehat{g}_{i j}=\delta \widehat{\psi}_{i} / \delta \psi^{j}$.

It must be emphasized that $\gamma_{\boldsymbol{q}}$ is a statistical property of the dynamics; it describes the mean growth rate of the $\mathrm{CC}$ energy spectrum after averaging over an ensemble of random DW's and random CC's. For homogeneous statistics, the correlation matrix $C_{\boldsymbol{k}}^{i j} \doteq\left\langle\psi_{\boldsymbol{k}}^{i} \psi_{\boldsymbol{k}}^{j *}\right\rangle$ obeys

$$
\partial_{t} \mathrm{C}_{\boldsymbol{k}}=\left[\left(\mathrm{L}_{\boldsymbol{k}}-\boldsymbol{\Sigma}_{\boldsymbol{k}}\right) \cdot \mathrm{C}_{\boldsymbol{k}}\right]^{H}+\cdots,
$$

where $H$ denotes the Hermitian part and the dots indicate omitted nonlinear-noise terms. ${ }^{3}$ The nonlinear mass operator $\boldsymbol{\Sigma}$ was discussed at length in Refs. 2 and 3. In non-Markovian statistical field theory, the procedure of Martin, Siggia, and $\operatorname{Rose}^{2-4}$ shows that $\boldsymbol{\Sigma}\left(t ; t^{\prime}\right)=$ $\delta \boldsymbol{G}(t) /\left.\delta\langle\boldsymbol{\psi}\rangle\left(t^{\prime}\right)\right|_{\hat{\boldsymbol{\eta}}=\mathbf{0}}$ (the semicolon denotes causality), where $\boldsymbol{G} \doteq\langle\widetilde{\boldsymbol{G}}\rangle, \widetilde{\boldsymbol{G}} \doteq[\mathrm{S} ; \widehat{\boldsymbol{\psi}}]$ (the divergence of a generalized flux; the semicolon implies matrix multiplication as well as the usual Poisson-bracket comma), and $\widehat{\boldsymbol{\eta}}(\boldsymbol{x}, t)$ is an arbitrary source added to the right-hand side of Eq. (3). Note that the variation is with respect to the mean field, and that after averaging over homogeneous statistics all mean fields vanish for $\widehat{\boldsymbol{\eta}}=\mathbf{0}$. The purpose of $\widehat{\boldsymbol{\eta}}$ is to break the symmetry, thereby allowing functional relationships between the statistical quantities of various orders ${ }^{3,4}$ to be uncovered. ${ }^{2}$ Further steps must be taken to reduce $\boldsymbol{\Sigma}\left(t ; t^{\prime}\right)$ to a Markovian form. The procedure involves the introduction of a tensor interaction time $\widehat{\boldsymbol{\theta}}$, as discussed in Ref. 2 for the scalar case.

$\gamma_{\boldsymbol{q}}$ is determined ${ }^{2}$ by $\boldsymbol{\Sigma}_{\boldsymbol{q}}^{H}$, which we now show can be obtained from second variation of an energy functional. The procedure involves the projection of the fluctuations into the DW $\left(\boldsymbol{\psi}^{\prime}\right)$ and $\mathrm{CC}(\underline{\boldsymbol{\psi}})$ subspaces [the underline means integrate over $z$; note that for any $A(\boldsymbol{x})$ one has $\bar{A}=\underline{\bar{A}}]$. Upon projecting Eq. (3), one obtains

$$
\begin{aligned}
\dot{\psi}^{\prime} & =-\left[\mathrm{S}^{\prime} ; \underline{\widehat{\boldsymbol{\psi}}}\right]-\left[\underline{\mathrm{S}} ; \widehat{\boldsymbol{\psi}}^{\prime}\right]-\left(\left[\mathrm{S}^{\prime} ; \widehat{\boldsymbol{\psi}}^{\prime}\right]-\underline{\widetilde{\boldsymbol{G}}}\right)+\widehat{\boldsymbol{\eta}}^{\prime}, \\
\dot{\boldsymbol{\psi}} & =-\underline{\widetilde{\boldsymbol{G}}}+\underline{\widehat{\boldsymbol{\eta}}},
\end{aligned}
$$

where $\underline{\widetilde{G}} \doteq \underline{\left[\mathrm{S}^{\prime} ; \widehat{\boldsymbol{\psi}}^{\prime}\right]}$ and tilde denotes a random function. (Some tildes are omitted to avoid clutter.) The shortwavelength energy $\widetilde{\mathcal{E}} \doteq \mathcal{H}\left[\boldsymbol{\psi}^{\prime}\right]$ obeys $\dot{\widetilde{\mathcal{E}}}=\overline{\widehat{\boldsymbol{\psi}}^{\prime} \cdot \dot{\boldsymbol{\psi}}^{\prime}}$, or

$$
\begin{aligned}
\dot{\tilde{\mathcal{E}}} & =-\overline{\widehat{\boldsymbol{\psi}}^{\prime} \cdot\left[\mathrm{S}^{\prime} ; \underline{\widehat{\psi}^{\prime}}\right]}-\overline{\widehat{\boldsymbol{\psi}}^{\prime} \cdot\left[\underline{\mathrm{S}} ; \widehat{\boldsymbol{\psi}}^{\prime}\right]}+\overline{\widehat{\boldsymbol{\psi}}^{\prime} \cdot \widehat{\boldsymbol{\eta}}^{\prime}} \\
& =\underline{\left[\mathrm{S}^{\prime} ; \widehat{\boldsymbol{\psi}}^{\prime}\right] \cdot \underline{\widehat{\boldsymbol{\psi}}}}-\operatorname{Tr} \overline{\left(\underline{\mathrm{S}} \cdot\left[\underline{\left.\widehat{\boldsymbol{\psi}}^{\prime}, \widehat{\boldsymbol{\psi}}^{\prime}\right]}\right)\right.}+\overline{\widehat{\boldsymbol{\psi}}^{\prime} \cdot \widehat{\boldsymbol{\eta}}^{\prime}} .
\end{aligned}
$$

The first term contains the definition of $\underline{\widetilde{\boldsymbol{G}}}$. The second term vanishes due to the antisymmetry of the Poisson bracket [cf. Eq. (4)]. Upon averaging Eq. (7b), noting that $\overline{\left\langle\boldsymbol{\psi}^{\prime}\right\rangle}=\overline{\left\langle\underline{\boldsymbol{\psi}^{\prime}}\right\rangle}=\mathbf{0}$, and defining $\underline{\boldsymbol{Q}} \doteq\langle\underline{\boldsymbol{\psi}}\rangle, \underline{\boldsymbol{P}} \doteq\langle\underline{\hat{\boldsymbol{\psi}}}\rangle$, $\underline{\boldsymbol{G}} \doteq\langle\underline{\widetilde{G}}\rangle$, and $\overline{\mathcal{E}} \doteq\langle\widetilde{\mathcal{E}}\rangle$, we are led to the generalized Poynting theorem $^{2} \dot{\overline{\mathcal{E}}} \approx \overline{\boldsymbol{G}} \cdot \underline{\boldsymbol{P}}$. (We neglected statistical correlations between $\underline{\widetilde{G}}$ and $\underline{\underline{\boldsymbol{\psi}}} .{ }^{2}$ ) The $\boldsymbol{x}$ dependences of $\underline{\boldsymbol{G}}$ and $\underline{\boldsymbol{P}}$ arise from the source $\widehat{\boldsymbol{\eta}}$; for $\widehat{\boldsymbol{\eta}}=\mathbf{0}$ and homogeneous statistics, $\underline{\boldsymbol{G}}, \underline{\boldsymbol{P}}$, and $\dot{\overline{\mathcal{E}}}$ all vanish.

For slowly varying $\mathrm{CC}$ quantities, we now write $(\boldsymbol{X}, T)$ instead of $(\boldsymbol{x}, t)$. Assuming Markovian statistics, we treat $T$ as a parameter; functionals are integrated only over the dummy integration variable $\overline{\boldsymbol{X}}$. Then

$$
\delta \dot{\overline{\mathcal{E}}} / \delta \underline{P}_{i}(\boldsymbol{X})=\underline{G}^{i}(\boldsymbol{X})+\underline{\Sigma}^{k i}(\overline{\boldsymbol{X}} ; \boldsymbol{X}) \cdot \underline{P}_{k}(\overline{\boldsymbol{X}}),
$$

where the contravariant CC mass operator is defined by $\underline{\Sigma}^{i k}\left(\boldsymbol{X} ; \boldsymbol{X}^{\prime}\right) \doteq \delta \underline{G}^{i}(\boldsymbol{X}) / \delta \underline{P}_{k}\left(\boldsymbol{X}^{\prime}\right)$. At second order we may differentiate with respect to either $\underline{\boldsymbol{Q}}$ or $\underline{\boldsymbol{P}}$. $\underline{\boldsymbol{Q}}$ produces a covariant index, as can be seen by noting that $\widehat{g}_{k j}=\delta \underline{P}_{k} / \delta Q^{j}$ and $\underline{\Sigma}^{k i} g_{k j}=\underline{\Sigma}_{j}{ }^{i}$. Upon Fourier transforming $\left(\boldsymbol{X}-\boldsymbol{X}^{\prime} \rightarrow \boldsymbol{q}\right)$, we obtain for $\widehat{\boldsymbol{\eta}}=\mathbf{0}$

$$
\frac{\delta^{2} \dot{\overline{\mathcal{E}}}}{\delta \underline{P}_{\boldsymbol{q}, i}^{*} \delta \underline{P}_{\boldsymbol{q}, j}}=2\left(\underline{\boldsymbol{\Sigma}}_{\boldsymbol{q}}^{H}\right)^{i j}, \frac{\delta^{2} \dot{\overline{\mathcal{E}}}}{\delta \underline{P}_{\boldsymbol{q}, i}^{*} \delta \underline{Q}_{\boldsymbol{q}}^{j}}=2\left(\underline{\boldsymbol{\Sigma}}_{\boldsymbol{q}}^{H}\right)^{i}{ }_{j},
$$

where $\boldsymbol{\Sigma}^{H} \doteq \frac{1}{2}\left(\boldsymbol{\Sigma}+\boldsymbol{\Sigma}^{\dagger}\right)$ and $\underline{\Sigma}_{j}^{i}(\boldsymbol{q})^{\dagger} \doteq \underline{\Sigma}_{j}{ }^{i}(\boldsymbol{q})^{*}$. The significance of $\underline{\boldsymbol{\Sigma}}^{H}$ can be understood by writing the nonlinear part of Eq. (5) for the CC's and lowering the second index to obtain $\partial_{t} \underline{C}_{j}^{i}=-2\left(\underline{\Sigma}^{i}{ }_{k} \underline{C}^{k}{ }_{j}\right)^{H}=-2\left(\underline{\Sigma}^{i k} \underline{C}_{k j}\right)^{H}$. Upon noting that $\underline{C}_{j}^{i}$ is Hermitian, one obtains the rate of change of the mean CC energy $\underline{\mathcal{E}}=\frac{1}{2} \underline{C}^{i}{ }_{i}$ as $\underline{\dot{\mathcal{E}}}=-\left(\underline{\Sigma}^{H}\right)^{i}{ }_{k} \underline{C}^{k}{ }_{i}=-\left(\underline{\Sigma}^{H}\right)^{i k} \underline{C}_{k i}$. Thus knowledge of $\underline{\underline{C}}$ and $\underline{\Sigma}^{H}$ completely determines $\gamma_{\boldsymbol{q}} \doteq \underline{\mathcal{E}}_{\boldsymbol{q}} / 2 \underline{\mathcal{E}}_{\boldsymbol{q}}$, e.g., ${ }^{15}$

$$
\begin{aligned}
\gamma_{\boldsymbol{q}} & =-\left[\left(\underline{\Sigma}_{\boldsymbol{q}}^{H}\right)^{i j} \underline{C}_{j i}(\boldsymbol{q})\right] / \underline{C}_{k}^{k}(\boldsymbol{q}) \\
& =-\frac{1}{2}\left(\frac{\delta^{2} \dot{\overline{\mathcal{E}}}}{\delta \underline{P}_{\boldsymbol{q}, i}^{*} \underline{P}_{\boldsymbol{q}, j}} \underline{C}_{j i}(\boldsymbol{q})\right) / \underline{C}_{k}^{k}(\boldsymbol{q}) .
\end{aligned}
$$

To find an explicit expression for $\dot{\overline{\mathcal{E}}}$ due to the CC's, one must generalize Eq. (5) to weakly inhomogeneous 
statistics. An extensive discussion of the scalar case was given in Ref. 2. Two-point observables are written as $A\left(\boldsymbol{x}, t, \boldsymbol{x}^{\prime}, t^{\prime}\right)=A(\boldsymbol{\rho}, \tau \mid \boldsymbol{X}, T)$, where $\boldsymbol{\rho} \doteq \boldsymbol{x}-\boldsymbol{x}^{\prime}$, $\boldsymbol{X} \doteq \frac{1}{2}\left(\boldsymbol{x}+\boldsymbol{x}^{\prime}\right), \tau \doteq t-t^{\prime}$, and $T \doteq \frac{1}{2}\left(t+t^{\prime}\right)$. Note that $\bar{A} \doteq \int d \boldsymbol{x} A(\boldsymbol{x}, \boldsymbol{x})=\sum_{\boldsymbol{k}} \int d \boldsymbol{X} A_{\boldsymbol{k}}(\boldsymbol{X})$, where the Fourier transform is with respect to $\boldsymbol{\rho}$. If under CC modulation the DW's evolve according to $\partial_{t} \boldsymbol{\psi}^{\prime}+i \widehat{\boldsymbol{\Omega}} \cdot \boldsymbol{\psi}^{\prime}=\mathbf{0}$, where $\widehat{\boldsymbol{\Omega}}$ is a linear operator (possibly a product of two noncommuting operators $\widehat{A}$ and $\widehat{B}$ ) whose Fourier transform is $\boldsymbol{\Omega}_{\boldsymbol{k}}(\boldsymbol{X})$, then the tensor WKE for the DW spectrum is

$\partial_{T} C_{\boldsymbol{k}}(\boldsymbol{X}, T)=2\left(\boldsymbol{\Omega}_{\boldsymbol{k}} \cdot \mathrm{C}_{\boldsymbol{k}}\right)^{A}-\left(\left\{\boldsymbol{\Omega}_{\boldsymbol{k}} ; \mathrm{C}\right\}+\left\{\mathrm{A}_{\boldsymbol{k}} ; \mathrm{B}_{\boldsymbol{k}}\right\} \cdot \mathrm{C}_{\boldsymbol{k}}\right)^{H}$,

where $\mathrm{M}^{A} \doteq(2 i)^{-1}\left(\mathrm{M}-\mathrm{M}^{\dagger}\right)$ and $\left\{A_{\boldsymbol{k}}, B_{\boldsymbol{k}}\right\} \doteq\left(\boldsymbol{\nabla} A_{\boldsymbol{k}}\right)$. $\left(\partial_{\boldsymbol{k}} B_{\boldsymbol{k}}\right)-\left(\partial_{\boldsymbol{k}} A_{\boldsymbol{k}}\right) \cdot\left(\boldsymbol{\nabla} B_{\boldsymbol{k}}\right)\left(\boldsymbol{\nabla} \equiv \partial_{\boldsymbol{X}}\right)$; one has $\overline{\left\{A_{\boldsymbol{k}}, B_{\boldsymbol{k}}\right\}}=$ 0 . (This new definition of a brace-delimited bracket should cause no confusion in context.) The first two terms of Eq. (6a) define $\boldsymbol{\Omega}_{\boldsymbol{k}}=\underline{\boldsymbol{\Omega}}_{\boldsymbol{k}}+\underline{\boldsymbol{\Omega}}_{k}^{\prime}$, where

$$
\begin{aligned}
\underline{\Omega}^{i}{ }_{k}[\underline{\boldsymbol{P}}] & \doteq-S^{i j}{ }_{k} \widehat{D} \underline{P}_{j}, \\
{\underline{\Omega^{\prime}}}^{i}{ }_{k}[\underline{\boldsymbol{Q}}] & \doteq(\underbrace{S^{i \bar{k}}{ }_{j} \widehat{D} \underline{Q}^{j}}_{\widehat{\mathrm{A}}})(\underbrace{g_{\bar{k} k}}_{\widehat{\mathrm{B}}}),
\end{aligned}
$$

and $\widehat{D} \doteq \boldsymbol{k} \cdot \widehat{\boldsymbol{z}} \times \boldsymbol{\nabla}$. We note the appearance of the operators $\widehat{\mathrm{A}}$ and $\widehat{\mathrm{B}}$, which do not commute because $\widehat{D}=$ $\widehat{D}_{\boldsymbol{k}}[\boldsymbol{\nabla}], \widehat{\mathrm{g}}=\widehat{\mathrm{g}}_{\boldsymbol{k}}[\boldsymbol{\nabla}]$, and $\underline{\boldsymbol{Q}}=\underline{\boldsymbol{Q}}_{\boldsymbol{k}}(\boldsymbol{X})$. The contribution from $\{\mathrm{A} ; \mathrm{B}\}=\boldsymbol{\nabla} \mathrm{A} \cdot \partial_{\boldsymbol{k}} \widehat{\mathrm{g}}$ adds to a part of $\left\{\underline{\boldsymbol{\Omega}^{\prime}} ; \mathrm{C}\right\}$ to give a term proportional to $\partial_{\boldsymbol{k}}\left(g_{k l} C^{l j}\right)=\partial_{\boldsymbol{k}} C_{k}{ }^{j}$. One obtains

$$
\begin{aligned}
\partial_{T} C^{i j}= & 2\left(\Omega_{k}^{i} C^{k j}\right)^{A} \\
& -\left(\left\{\underline{\Omega}^{i}{ }_{k}[\underline{\boldsymbol{P}}], C^{k j}\right\}+\left\{\underline{\Omega}^{\prime i k}[\underline{\boldsymbol{Q}}], C_{k}{ }^{j}\right\}\right)^{H} .
\end{aligned}
$$

The $\underline{\Omega}^{\prime}$ term is small and will be neglected. The resulting modulated Eq. (13) conserves the same Casimirs as do the primitive dynamics. That is expected since Casimir invariance is independent of the Hamiltonian.

To determine $\dot{\overline{\mathcal{E}}}$, we lower the second index of Eq. (13) with $g_{\bar{\jmath} j}$ and take the trace. We write the resulting equation in conservative form by passing $g_{\bar{\jmath} j}$ through the Poisson brackets, obtaining correction terms ${ }^{2}$ that combine to an antisymmetric form that vanishes under the trace. Next, we bar the equation and vary $\dot{\overline{\mathcal{E}}}$ according to Eq. (9). On the right-hand side, we calculate $\delta \mathrm{C} / \delta \underline{\boldsymbol{P}}$ from the steady-state form of Eq. (13) (the omitted linear and nonlinear DW dynamics contribute a term $\widehat{\boldsymbol{\theta}}^{-1}: \mathrm{C}$, where the fourth-rank tensor $\widehat{\boldsymbol{\theta}}$ generalizes the triad interaction time of scalar Markovian theory $\left.{ }^{2}\right)$. The final result is $\left(\Sigma^{i j}\right)^{H}=H^{(i j)}\left(\Sigma^{i j}\right)$, where $H^{(i j)}$ denotes the Hermitian part with respect to the indices $i$ and $j$, and

$$
\begin{aligned}
\Sigma_{\boldsymbol{q}}^{i j}=-\sum_{\boldsymbol{k}} d^{2} S^{i r}{ }_{k}\left[(\partial+2 i) g_{r s}\right] & \\
& \times \widehat{\boldsymbol{\theta}}^{k s}{ }_{k \bar{s}} H^{(\bar{k} \bar{s})}\left[S^{j \bar{k}}{ }_{l}(\partial+2 i) C_{\boldsymbol{k}}^{l \bar{s}}\right],
\end{aligned}
$$

$d \doteq \widehat{\boldsymbol{z}} \cdot \boldsymbol{q} \times \boldsymbol{k}$, and $\partial \doteq \boldsymbol{q} \cdot \partial_{\boldsymbol{k}}$. The terms proportional to $2 i$ are associated with off-diagonal correlations and vanish in the scalar case. Note that whereas only $\boldsymbol{\Sigma}^{H}$ is determined from the energy theorem, WKB analysis of $\boldsymbol{G}$ itself $^{6}$ can be shown to lead directly to Eq. (14). The form (14) is not unique; for each Casimir $\mathcal{Z}^{(n)}$, one element of $C^{i j}$ could be eliminated in favor of $\mathcal{Z}^{(n)}$.

As a nontrivial application, we first consider electrostatic ITG fluctuations at small but nonzero $\tau .{ }^{16}$ The relevant gyrofluid equations were well studied in Refs. 9 11, although their Hamiltonian structure has not been previously examined. Let $\boldsymbol{\psi}=(N, T)^{T}$, where $N$ and $T$ are the fluctuations in ion gyrocenter density and temperature, respectively. For $\tau \ll 1$, the gyrokinetic-Poisson equation is $N=\alpha \varphi-\left(1+\frac{1}{2} \tau \alpha\right) \omega-\frac{1}{2} \tau \nabla_{\perp}^{2} T$, where $\omega \doteq \nabla_{\perp}^{2} \varphi$ and $\alpha$ projects onto the DW subspace. Let

$$
\mathcal{H}[\boldsymbol{\psi}]=\frac{1}{2} \overline{\left(N+\frac{1}{2} \tau \nabla_{\perp}^{2} T\right) \widehat{K}\left(N+\frac{1}{2} \tau \nabla_{\perp}^{2} T\right)},
$$

where $\widehat{K} \doteq\left(1+\frac{1}{2} \tau \nabla_{\perp}^{2}\right)\left[\alpha-\left(1+\frac{1}{2} \tau \alpha\right) \nabla_{\perp}^{2}\right]^{-1}[$ note $\widehat{K}(N+$ $\left.\left.\frac{1}{2} \tau \nabla_{\perp}^{2} T\right)=\varphi+\frac{1}{2} \tau \omega\right]$. The metric tensor and structure matrix are

$$
\widehat{\mathrm{g}}=\left(\begin{array}{cc}
1 & \widehat{b} \\
\widehat{b} & \widehat{b}^{2}
\end{array}\right) \widehat{K}, \quad \mathrm{~S} \doteq\left(\begin{array}{cc}
N & T \\
T & N+T
\end{array}\right),
$$

where $\widehat{b} \doteq \frac{1}{2} \tau \nabla_{\perp}^{2}$. S satisfies $S^{i j}{ }_{k}=S^{i k}{ }_{j}$. One obtains

$$
\begin{aligned}
\dot{N} & =-\left[\varphi+\frac{1}{2} \tau \omega, N\right]-\frac{1}{2} \tau\left[\omega+\underline{\frac{1}{2} \tau \nabla_{\perp}^{2} \omega}, T\right], \\
\dot{T} & =-\left[\varphi+\frac{1}{2} \tau \omega, T\right]-\frac{1}{2} \tau\left[\omega+\underline{\frac{1}{2} \tau \nabla_{\perp}^{2} \omega}, N+T\right] .
\end{aligned}
$$

This system conserves $\mathcal{H}=\overline{\varphi^{\prime 2}}+\overline{|\nabla \varphi|^{2}}-\frac{1}{2} \tau \overline{\omega^{2}}-\frac{1}{4} \tau^{2} \overline{\omega^{\prime 2}}$ and the Casimir $\mathcal{Z} \doteq \frac{1}{2}\left(\overline{N^{2}}+\overline{T^{2}}\right)$. If all terms of $O(\tau)$ are neglected, Eq. (17a) reduces to the generalized Hasegawa-Mima equation. ${ }^{2}$ In that limit, it can be readily verified that formula (14) reduces to the result of KK for that case; this is a nontrivial cross-check.

The underlined terms are $O\left(\tau^{2}\right)$; if only they are neglected, the resulting system is the symmetrical one studied in Refs. 9-11. ${ }^{17}$ As in Ref. 5, one might attempt to calculate the $O(\tau)$ correction to $\gamma_{\boldsymbol{q}}$. However, there is a fundamental reason why this is impossible within this (and all similar) formalism: the $O(\tau)$ changes in C and $\widehat{\boldsymbol{\theta}}$ due to self-consistency are unknown. If one were to asystematically evaluate those functions at $\tau=0$, one would proceed as follows. The dominant terms in formula (14) are $\gamma_{\boldsymbol{q}} \approx-\underline{g}_{N N}(\boldsymbol{q}) \underline{\Sigma}_{\boldsymbol{q}}^{N N}$, where $\underline{\Sigma}_{\boldsymbol{q}}^{N N} \approx$ $-\frac{1}{2} \sum_{\boldsymbol{k}} d^{2}\left(\partial g_{N N} \widehat{\boldsymbol{\theta}}_{r}^{N N} \partial C^{N N}+2 \partial g_{N T} \widehat{\boldsymbol{\theta}}_{r}^{N T} \partial C_{r}^{N T}\right), r$ denotes the real part, and $\widehat{\boldsymbol{\theta}}$ is taken to be diagonal. (This assumption may be problematical; see the remarks in the next paragraph.) Dependence on $\tau$ occurs in several places: (i) $\underline{g}_{N N}(\boldsymbol{q}) \approx q^{-2}$; (ii) the derivatives $-\partial g_{N N}(\boldsymbol{k}) \approx 2(\boldsymbol{q} \cdot \boldsymbol{k})(1+\tau)\left[1+\left(1+\frac{1}{2} \tau\right) k^{2}\right]^{-2}$ and $-\partial g_{N T}(\boldsymbol{k}) \approx \tau(\boldsymbol{q} \cdot \boldsymbol{k})\left(1+k^{2}\right)^{-2}$. The term in $C^{N N}$ varies as $1+\tau$ for $k^{2} \ll 1$, while the term in $C^{N T}$ varies as $\tau \operatorname{sgn}\left(\widehat{q} \cdot \partial_{\boldsymbol{k}} \operatorname{Re} C^{N T} / \widehat{q} \cdot \partial_{\boldsymbol{k}} C^{N N}\right)$. Thus the sign of the cross-correlation is crucial. In linear theory, one finds $T=\left\{\omega_{*}^{T} /\left[\left(1+k^{2}\right) \omega_{r}\right]-1\right\} N$, where $\omega_{*}$ is the diamagnetic frequency and $\omega_{r}$ is the linear mode frequency. This 
is typically negative. However, we must stress again that the ultimate $\tau$ dependence cannot be ascertained without a self-consistent theory of the steady-state $C$ and $\widehat{\boldsymbol{\theta}}$.

Next we consider collisional DW fluctuations at small plasma pressure $\beta$. With $\boldsymbol{\psi}=(\omega, n, A)^{T}$, where $n$ is the electron density and $A$ is the parallel component of the vector potential, the nonlinear parts of the equations of Ref. 12 are generated by $\mathcal{H}[\boldsymbol{\psi}]=\frac{1}{2} \int d \boldsymbol{x}\left[\omega\left(-\nabla^{-2}\right) \omega+n^{2}+\right.$ $\left.\beta A\left(-\nabla^{2}\right) A\right]$ and $\mathrm{S}=\left(\begin{array}{ccc}\omega & n & A \\ n & n & A \\ A & A & 0\end{array}\right)$. Casimir invariants are $\mathcal{Z}^{(1)} \doteq \frac{1}{2} \overline{(n-\omega)^{2}}$ and $\mathcal{Z}^{(2)} \doteq \frac{1}{2} \overline{A^{2}}$. As a nontrivial crosscheck, it can be shown that in the collisionless and $\beta \rightarrow 0$ limit one recovers the generalized HM result of KK. This limit is subtle, as formula (14) with diagonal $\widehat{\boldsymbol{\theta}}$ presents the result in terms of $\partial C^{\omega \omega}$, not $\partial \mathcal{Z}^{(1)}$. The resolution is that $\widehat{\boldsymbol{\theta}}$ cannot be considered to be diagonal because of the rigid constraint engendered by adiabatic DW response; rather, it reduces to the product of a scalar interaction time and a singular nondiagonal tensor. For fixed $\mathrm{C}$ and $\widehat{\boldsymbol{\theta}}$, a formula for the $O(\beta)$ correction to the electrostatic $\gamma_{\boldsymbol{q}}$ can be written straightforwardly, aided by the facts that $\widehat{\mathrm{g}}$ is diagonal and $\partial g_{n n}=0$. However, the expression is somewhat lengthy and is furthermore not unique because of the Casimir constraints; we have been unable to ascertain a definite sign. In any event, the true $\beta$ dependence cannot be determined without a selfconsistent analysis of the steady-state turbulence. Further details and discussion will be presented elsewhere. ${ }^{13}$

In summary, the convective-cell growth rate $\gamma_{\boldsymbol{q}}$ is fundamentally a nonlinear quantity; ${ }^{2}$ linear theory enters only indirectly through the values of the triad interac- tion time $\widehat{\boldsymbol{\theta}}$ and the wave-number spectrum $C_{\boldsymbol{k}}$. Thus it is useful to base the choice of dependent variables on the nonlinear structure of the primitive equations. Accordingly, we have developed the theory of $\gamma_{\boldsymbol{q}}$ in terms of a Hamiltonian functional description in noncanonical coordinates. A Hamiltonian of generalized kinetic-energy form specified by the covariant tensor $\widehat{\mathrm{g}}$ together with a certain Lie-Poisson bracket is sufficient to reproduce various popular Eulerian gyrofluid systems. The dynamics evolve on the symplectic leaf specified by the Casimir invariant(s) $\mathcal{Z}$. $\widehat{g}$ serves as a metric tensor to lower contravariant indices. The tensor wave kinetic equation has a natural covariant form. $\gamma_{\boldsymbol{q}}$ is given in terms of the $g_{i j}$, the structure constants $S^{i j}{ }_{k}$, and the spectral functions $C^{i j}$ according to formulas (10b) and (14). The general expression reduces to the known result for generalized Hasegawa-Mima dynamics, ${ }^{2,3}$ and more elaborate coupled systems can also be analyzed systematically.

Important questions remain unanswered. The formalism does not determine $\widehat{\boldsymbol{\theta}}$ or the nonlinear phase relations in steady-state turbulence. Toroidal geometry requires additional discussion. If no separation between long and short scales can be made, use of the wave kinetic equation must be replaced by more general statistical closure theory. ${ }^{2}$ It is hoped that the asymptotic limit studied here will serve as a useful benchmark for such analysis.

We are grateful for eludicating discussions with G. Hammett (on the nature of gyrofluid closures), P. Morrison (on Hamiltonian bracket formalisms), and B. Scott (on the importance of symmetrically conservative fluid systems). This work was supported by U. S. Dept. of Energy Contract No. DE-AC02-76-CHO-3073.
* Electronic address: krommes@princeton.edu

1 P. H. Diamond, M. N. Rosenbluth, F. L. Hinton, M. Malkov, J. Fleischer, and A. Smolyakov, 17th IAEA Fusion Energy Conference (International Atomic Energy Agency, Vienna, 1998), IAEA-CN-69/TH3/1.

2 J. A. Krommes and C.-B. Kim, Phys. Rev. E 62, 8508 (2000).

3 J. A. Krommes, Phys. Rep. 360, 1 (2002).

4 P. C. Martin, E. D. Siggia, and H. A. Rose, Phys. Rev. A 8, 423 (1973).

5 E.-j. Kim and P. H. Diamond, Phys. Plasmas 9, 4530 (2002).

6 J. A. Krommes, Comments on "Dynamics of zonal flow saturation in strong collisionless drift wave turbulence" [Phys. Plasmas 9, 4530 (2002)], Phys. Plasmas (2003) (in press).

7 P. J. Morrison, Rev. Mod. Phys. 70, 467 (1998).

8 A. I. Smolyakov and P. H. Diamond, Phys. Plasmas 6, 4410 (1999).

${ }^{9}$ W. Dorland and G. W. Hammett, Phys. Fluids B 5, 812 (1993).

10 M. A. Beer, Ph.D. thesis, Princeton University, 1995.

11 M. A. Beer and G. W. Hammett, Phys. Plasmas 3, 4046 (1996).

12 S. J. Camargo, B. D. Scott, and D. Biskamp, Phys. Plas- mas 3, 3912 (1996)

13 R. A. Kolesnikov and J. A. Krommes, Bull. Am. Phys. Soc. 48(9), 73 (2003).

14 It is possible that $\operatorname{det} \mathrm{g}_{k}=0$, so $\mathrm{g}_{k}$ need not be invertible in the conventional sense. However, it is never necessary to raise an index that has not previously been lowered with $\widehat{\mathrm{g}}$; thus, $s_{i}=\widehat{g}_{i j} \psi^{j}$ can always be solved.

15 That $\gamma_{\boldsymbol{q}}$ is determined by $\boldsymbol{\Sigma}^{H}$. C rather than just $\boldsymbol{\Sigma}^{H}$ is intimately related to the form of the Onsager symmetry matrix; see J. A. Krommes and G. Hu, Phys. Fluids B 5, 3908 (1993).

16 A calculation of $\gamma_{\boldsymbol{q}}$ for this limit was first attempted by KD. ${ }^{5}$ However, their algebra was tedious and they used asymmetrical equations that omitted some terms of $O(\tau){ }^{6}$

17 Beer $^{10}$ observed that $\mathcal{Z}$ was conserved through $O(\tau)$. The physical equations at $O\left(\tau^{2}\right)$ may differ from Eqs. (17). Kim and Diamond, ${ }^{5}$ following the original work of Horton, Estes, and Biskamp [Plasma Phys. 22, 663 (1980)], neglected all terms of $O(\tau)$ in the equation for $P=N+T$. The $\mathcal{E}$ for the resulting asymmetrical system differs at $O(\tau)$ from the one given by Eq. (15), as does $\mathcal{Z}$. [Use of $\partial_{t} P \approx-[\varphi, P]$ in Eq. (17a) in conjunction with the approximate relation $N \approx \alpha \varphi-\nabla^{2} \varphi-\frac{1}{2} \tau \nabla^{2} P$ leads to Eq. (27) of KD. That equation remains consistent through $O(\tau)$. 
However, the $\dot{P}$ equation itself is inconsistent.] 


\section{External Distribution}

Plasma Research Laboratory, Australian National University, Australia

Professor I.R. Jones, Flinders University, Australia

Professor João Canalle, Instituto de Fisica DEQ/IF - UERJ, Brazil

Mr. Gerson O. Ludwig, Instituto Nacional de Pesquisas, Brazil

Dr. P.H. Sakanaka, Instituto Fisica, Brazil

The Librarian, Culham Laboratory, England

Mrs. S.A. Hutchinson, JET Library, England

Professor M.N. Bussac, Ecole Polytechnique, France

Librarian, Max-Planck-Institut für Plasmaphysik, Germany

Jolan Moldvai, Reports Library, Hungarian Academy of Sciences, Central Research Institute for Physics, Hungary

Dr. P. Kaw, Institute for Plasma Research, India

Ms. P.J. Pathak, Librarian, Institute for Plasma Research, India

Ms. Clelia De Palo, Associazione EURATOM-ENEA, Italy

Dr. G. Grosso, Instituto di Fisica del Plasma, Italy

Librarian, Naka Fusion Research Establishment, JAERI, Japan

Library, Laboratory for Complex Energy Processes, Institute for Advanced Study, Kyoto University, Japan

Research Information Center, National Institute for Fusion Science, Japan

Dr. O. Mitarai, Kyushu Tokai University, Japan

Dr. Jiangang Li, Institute of Plasma Physics, Chinese Academy of Sciences, People's Republic of China

Professor Yuping Huo, School of Physical Science and Technology, People's Republic of China

Library, Academia Sinica, Institute of Plasma Physics, People's Republic of China

Librarian, Institute of Physics, Chinese Academy of Sciences, People's Republic of China

Dr. S. Mirnov, TRINITI, Troitsk, Russian Federation, Russia

Dr. V.S. Strelkov, Kurchatov Institute, Russian Federation, Russia

Professor Peter Lukac, Katedra Fyziky Plazmy MFF UK, Mlynska dolina F-2, Komenskeho Univerzita, SK-842 15 Bratislava, Slovakia

Dr. G.S. Lee, Korea Basic Science Institute, South Korea

Institute for Plasma Research, University of Maryland, USA

Librarian, Fusion Energy Division, Oak Ridge National Laboratory, USA

Librarian, Institute of Fusion Studies, University of Texas, USA

Librarian, Magnetic Fusion Program, Lawrence Livermore National Laboratory, USA

Library, General Atomics, USA

Plasma Physics Group, Fusion Energy Research Program, University of California at San Diego, USA

Plasma Physics Library, Columbia University, USA

Alkesh Punjabi, Center for Fusion Research and Training, Hampton University, USA

Dr. W.M. Stacey, Fusion Research Center, Georgia Institute of Technology, USA

Dr. John Willis, U.S. Department of Energy, Office of Fusion Energy Sciences, USA

Mr. Paul H. Wright, Indianapolis, Indiana, USA 
The Princeton Plasma Physics Laboratory is operated by Princeton University under contract with the U.S. Department of Energy.

\author{
Information Services \\ Princeton Plasma Physics Laboratory \\ P.O. Box 451 \\ Princeton, NJ 08543
}

Phone: 609-243-2750

Fax: 609-243-2751

e-mail: pppl_info@pppl.gov

Internet Address: http://www.pppl.gov 